\title{
Long-term survival in a recipient of kidney transplant without maintenance immunosuppression: A case report
}

\author{
SUJUAN FENG ${ }^{1}$, YUAN ZHUANG ${ }^{2}$, HANG LIU $^{1}$ and XIAODONG ZHANG ${ }^{1}$ \\ ${ }^{1}$ Institute of Uro-Nephrology, Beijing Chao-Yang Hospital, Capital Medical University, Beijing 100020; \\ ${ }^{2}$ Department of Blood Transfusion, Chinese PLA General Hospital, Beijing 100853, P.R. China
}

Received May 17, 2016; Accepted January 15, 2018

DOI: $10.3892 /$ etm.2018.6176

\begin{abstract}
Operational tolerance of an allograft kidney is the optimal condition in patients following kidney transplantation and recipient-mediated immunological rejection still remains the primary challenge following transplantation. In the present study, a case of long-term survival is reported in a patient following kidney transplantation without maintenance immunosuppression therapies for $>10$ years. Although the pathological conditions of the transplanted kidney revealed a degree of impaired kidney function, the patient exhibited a serum creatinine level of $119.2 \mu \mathrm{mol} / 1$ and no significant changes were observed in $\mathrm{T}$ or $\mathrm{B}$ cell biomarkers associated with immunologic tolerance. Notably, the patient's allograft was functioning normally. Consequently, further studies may need to elucidate the development of operational tolerance.
\end{abstract}

\section{Introduction}

Although kidney transplantation is currently considered as the preferred therapy for end-stage renal disease, morbidity induced by the use of immunosuppressive regimens and recipient-mediated immunological rejection remain the primary challenge following transplantation (1). It has previously been demonstrated that graft function may be affected by immunologic and non-immunologic factors, including race, height, age, sex and weight (2). Although the application of immunosuppressive agents is able to reduce pathological autoimmune reactions, it may also interfere with normal immune response, thus increasing the incidence of side effects, such as cardiovascular diseases, infection, malignancies, diabetes and other metabolic disorders $(3,4)$. Therefore, the development of novel immune tolerance protocols is required for transplantation, so that the long-term

Correspondence to: Dr Xiaodong Zhang, Institute of Uro-Nephrology, Beijing Chao-Yang Hospital, Capital Medical University, 8 Gong Ti Nan Lu, Chaoyang, Beijing 100020, P.R. China E-mail: drxdzhang@163.com

Key words: kidney transplantation, immunosuppression, operational tolerance immunosuppression induced by currently used agents does not occur. However, the mechanisms associated with the induction and maintenance of immune tolerance remain elusive. The present case report presents one case of potential immunological tolerance following kidney transplantation from a living donor and transient immunosuppressive therapy.

\section{Case report}

A 58-year-old female patient with end-stage renal disease secondary to chronic nephritis underwent living-donor kidney transplantation at the 251st Hospital of People's Liberation Army of China (Hebei, China) in February 2005. The kidney was donated by the patient's sister with $\mathrm{ABO}$ compatibility (blood type of donor and recipient were Type B) and human leukocyte antigen (HLA) incompatibilities (2/6 mismatch of HLA typing). De novo donor-specific antibodies were negative. Immunosuppression was performed following transplantation via a standard method including FK506 $(0.1 \mathrm{mg} / \mathrm{kg} / \mathrm{day})$, mycophenolate mofetil $(1,000 \mathrm{mg} / \mathrm{kg} / \mathrm{day})$ and prednisone for five days $(500 \mathrm{mg} / \mathrm{kg} / \mathrm{day})$, and normal graft function was observed 3 days following transplantation. However, due to the side effects of the immunosuppressive therapy, including thoracalgia and diarrhea, 7 days following transplantation, the patient refused to take mycophenolate mofetil capsules on the 8th day of treatment and the dosage of FK506 was decreased to $2 \mathrm{mg}$ per day, which resulted in hypouresis, edema and ascites. Therefore, the patient was obliged to start and maintain hemodialysis 40 days following kidney transplantation.

Following hemodialysis for two months, the patient's urine output increased to $500 \mathrm{ml} /$ day; therefore, she stopped using all immunosuppressive agents and subsequently stopped hemodialysis five months later. Notably, graft function subsequently normalized with a baseline serum creatinine level of $170-180 \mu \mathrm{mol} / 1$ (normal range, 57-97 $\mu \mathrm{mol} / 1$ ), accompanied by urine output increasing to $1,200 \mathrm{ml} /$ day (normal range, $1,000-3,000 \mathrm{ml} /$ day) $(5,6)$. In the last decade, the patient's graft function has been relatively stable, except for occasional body chills, joint pain (suspected rheumatoid arthritis) and renal pain.

The patient first attended Beijing Chao-Yang hospital (Beijing, China) for consultation as she often experienced pain around the area of transplanted kidney in the last four years. The medical history showed that her blood pressure was stable, 
at $\sim 130 / 90 \mathrm{mmHg}$. She seldom had a fever, although laboratory tests indicated the presence of urinary tract infection [white blood cell (WBC) count, 287.7/ $\mu$ l; bacteria count, 18,996.1/ $\mu 1]$. The serum creatinine level was $119.2 \mu \mathrm{mol} / \mathrm{l}$ and urea nitrogen was $8.66 \mu \mathrm{mol} / 1$, whereas a high cystatin $\mathrm{C}$ level was observed $(2.24 \mathrm{ng} / \mathrm{l})$. Negative results were obtained for panel reactive antibodies class I and II screening, and 24-h urinary protein quantity was $88 \mathrm{mg}$. The level of fasting blood-glucose was $5.46 \mathrm{mmol} / 1$, whereas glycated albumin and glycosylated hemoglobin were 16.7 and $7.2 \%$, respectively. Other laboratory findings, including cholesterol, glutamic-oxalacetic transaminease, glutamic-pyruvic transaminase and total bilirubin were normal.

$\mathrm{T}$ cells were isolated from peripheral blood mononuclear cells and determined by flow cytometry. A total of $5 \times 10^{7} / \mathrm{ml}$ peripheral blood mononuclear cells (PBMCs) were isolated from freshly heparinized whole blood by standard Ficoll density gradient centrifugation (room temperature; $800 \mathrm{x} \mathrm{g}$ for $30 \mathrm{~min}$; GE Healthcare, Chicago, IL, USA). To determine the frequency and phenotype of myeloid derived suppressor cell; cluster of differentiation (CD) 4 cells, CD8 cells, $\mathrm{T}$ regulatory and nature killer cells in PBMCs, multicolor fluorescence-activated cell staining analysis was performed using the following antibodies sourced from BD Pharmingen; BD Biosciences (Franklin Lakes, NJ, USA): anti-CD3 (cat. no. 557832; 1:10), anti-CD4 (cat. no. 562281; 1:10), anti-CD8 (cat. no. 555366; 1:10), anti-CD14 (cat. no. 557832; 1:10), anti-HLA DR (cat. no. 555812; 1:10), anti-CD11b (cat. no. 340937; 1:10), anti-CD56 (cat. no. 556647; 1:10), anti-CD33 (cat. no. 555450; 1:10), anti-CD34 (cat. no. 550761; 1:10), anti-CD25 (cat. no. 560990; 1:10), anti-CD127 (cat. no. 557938; 1:10), anti-CD19 (cat. no. 555415; 1:10), anti-CD20 (cat. no. 563780; 1:10) and anti-forkhead box P3 (FOXP3 1:10; cat. no. 77-5776-40; eBioscience; Thermo Fisher Scientific, Inc., Waltham, MA, USA). For surface marker staining, PBMCs were incubated with the appropriate antibodies following three gentle washes with PBS. For intracellular marker staining, PBMCs were washed with PBS, then fixed and permeabilized with Cytofix/Cytoperm solution on ice for $1 \mathrm{~h}$ (BD Pharmingen; BD Biosciences), anti-FOXP3 antibodies were utilized for intracellular staining by incubating with PBMCs at $4^{\circ} \mathrm{C}$ for $30 \mathrm{~min}$ in the dark. Flow cytometry was performed using a Beckman Coulter FC500 MPL (Beckman Coulter, Inc., Brea, CA, USA). Analysis of FACS-data was completed using FlowJo software 7.6.3 (Tree Star, Inc., Ashland, OR, USA). Isotype-matched antibodies were used with all the samples as controls. The CD3+ T cell count was $1,054 / \mu 1$ (59.4\%), CD3+CD4+ was 643/ $\mu 1(33 \%)$ and CD3+CD8+ was $391 / \mu 1(22.1 \%)$. The proportions of regulatory B cells, T cells, suppressor $\mathrm{T}$ cells and nature killer cells were 12.0, 3.0, 13.4 and $12.9 \%$, respectively. The proportion of myeloid-derived suppressor cells was $1 \%$, which was within the normal range $(1-2 \%)(7)$.

Spiral abdominal computed tomography revealed that two native kidneys were atrophied and that the size, shape and density of the transplanted kidney, which was located on the right iliac fossa, showed no marked abnormalities (Fig. 1).

Color Doppler ultrasonography examination revealed that the size of the transplanted kidney was $9.9 \times 4.8 \mathrm{~cm}$, the thickness of kidney parenchyma was $1.6 \mathrm{~cm}$, the blood supply

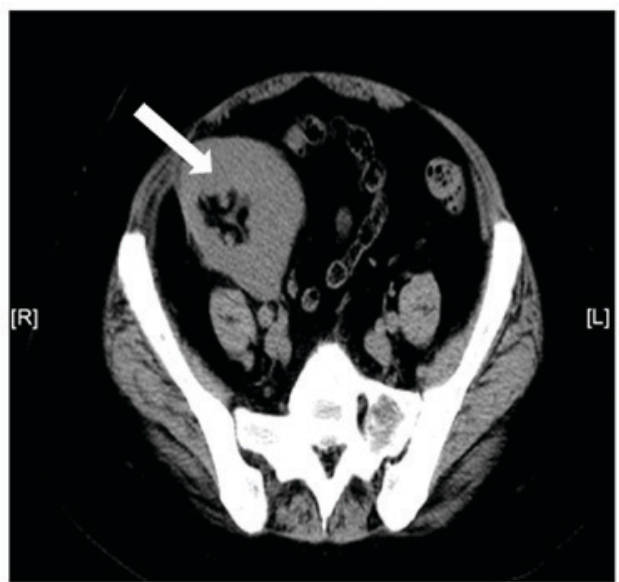

Figure 1. Spiral abdominal computed tomography image demonstrating the location of the kidney allograft (white arrow) laterally in the right lower quadrant and the atrophy of two native kidneys, which are almost invisible.

of transplanted kidney was good and there was no pelviectasis; however, kidney artery blood flow resistance index had increased to $0.74-0.78$ (Fig. 2).

Graft biopsy for this patient was performed twice by nephrocentesis under guidance of ultrasound, as the initial pathological findings were contrary to the patient's clinical manifestation. The result revealed ischemic sclerosis in the glomerulus, the kidney tubular epithelial cells were flat and a number of kidney tubules were atrophied. Protein cast, cell cast and inflammatory cell infiltration were also observed in some kidney tubules. Kidney interstitial hyperplasia was also observed (Fig. 3A). To further confirm the pathological conditions of the transplanted kidney, graft biopsy was conducted for the second time. The results demonstrated few glomerulus ischemic sclerosis, partial renal tubular dilatation and infiltration of inflammatory cells (Fig. 3B). The patient was in stable condition without other dominant diseases.

The present study was approved by the Ethics Committee of Beijing Chao-Yang Hospital (Capital Medical University, Beijing, China). Written informed consent form was obtained from the patient. All procedures performed were in accordance with the ethical standards of the institutional and/or national research committee and with the 1964 Helsinki declaration and its later amendments or comparable ethical standards.

\section{Discussion}

Following the discovery of immunologic tolerance by Ray Owen in 1945, who observed that placental interchange resulted in red cell chimerism between dizygotic bovine twins (8), there has been a great effort to establish tolerance through various different strategies. The original definition of tolerance was provided by Billingham et al in the 1950s (9), which referred to non-responsiveness to antigens. At present, tolerance is categorized in three subtypes: Central tolerance, which is mediated by transplanted donor hematopoietic cells and concerns the recognition of donor antigens as self-antigens; peripheral tolerance, which is not inducted by hematopoetic cell transplantation, but by either 
A

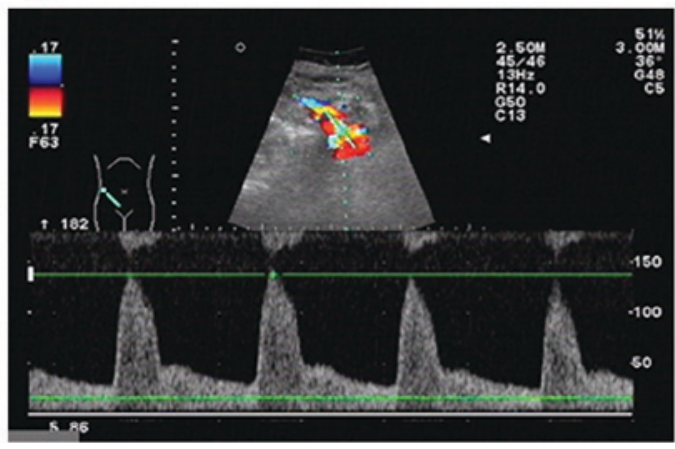

B

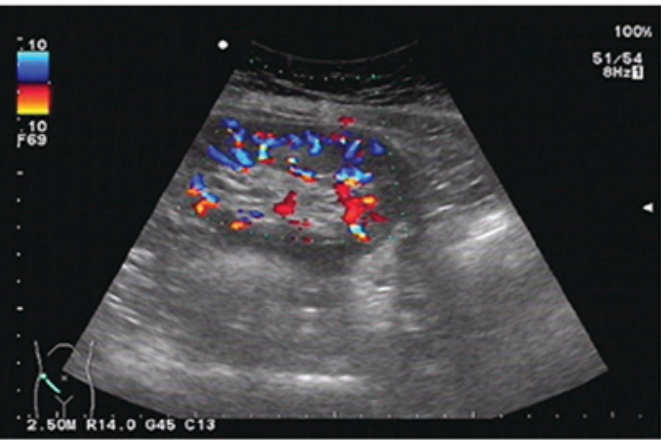

Figure 2. Doppler ultrasonography demonstrated the blood supply of transplanted kidney. (A) Duplex Doppler waveform showed high systolic arterial peaks with normal diastolic flow and high resistance arterial flow. (B) Longitudinal color Doppler image showed relatively abundant blood flow and normal size of transplanted kidney.

A

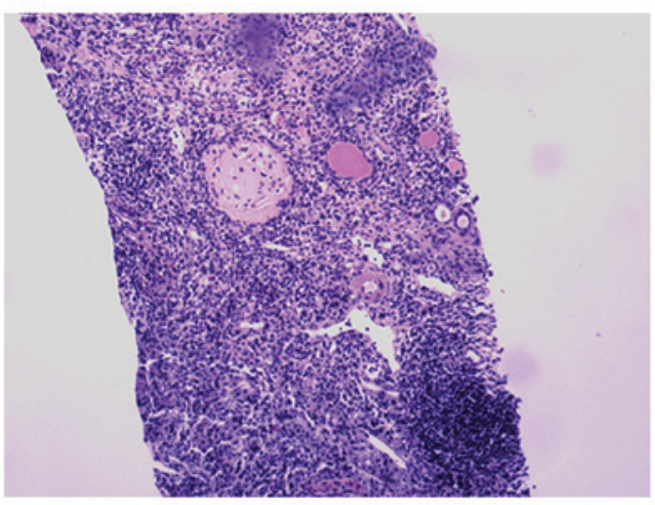

B

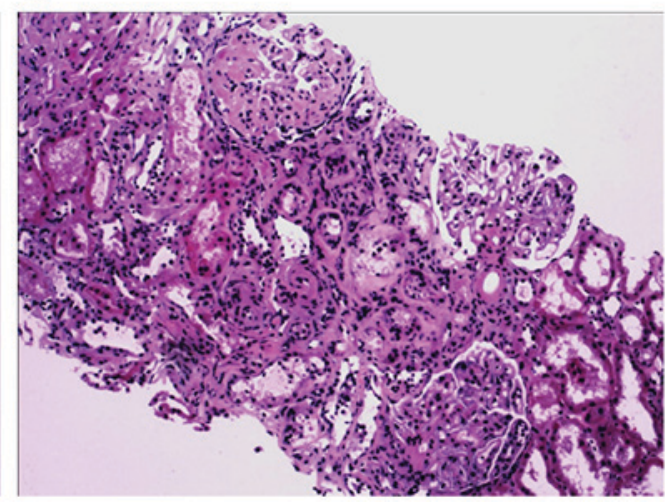

Figure 3. H\&E staining of renopuncture biopsy. (A) H\&E staining of the initial renopuncture biopsy indicated ischemic sclerosis of glomerulus, flat kidney tubular epithelial cells, atrophy of some kidney tubules and interstitial hyperplasia of transplanted kidney. (B) H\&E staining of the second renopuncture biopsy indicated reduced glomerulus ischemic sclerosis, renal tubular dilatation and infiltration of inflammatory cells than the results of the initial examination. H\&E, hematoxylin and eosin.

pharmacological immunosuppressive agents or biological agent-led deletion or suppression of self-reactive $\mathrm{T}$ cells; and operational tolerance, which is typically observed in patients who stopped immunosuppressive therapy for at least one year and in whom no destructive alloimmune response was observed (10). Notably, operational tolerance was more often observed following organ transplantation. Although kidney transplant recipients were prone to rejection following immunosuppressive withdrawal, $>200$ cases of operational tolerance persisting for at least one year have been reported (11). Therefore, elucidation of the operational tolerance-related molecular mechanisms and biomarkers may identify novel targets for kidney transplantation therapy.

The molecular basis of operational tolerance have been analyzed by gene-expression profiling in kidney transplant recipients, in which various genes, including tumor growth factor- $\beta$ in the blood of kidney transplant recipients, were demonstrated to be minimally invasive monitoring tools for guiding operational tolerance titration (12). In 25 operational tolerant kidney transplant recipients, 30 differentially-expressed genes were identified to be specific for B cells compared with those patients receiving immunosuppression by using peripheral blood microarrays (13). Increases of regulatory $\mathrm{T}$ cells were found in the maintenance of immunologic tolerance by suppressing aberrant or excessive immune responses (14). In addition, patients with operational tolerance were also characterized by a higher number of B cells in the blood compared with patients with stable graft function under immunosuppression and patients with antibody-mediated chronic rejection (15). Therefore, strategies for operational tolerance including the pharmacological, biological and cellular therapies were categorized by $\mathrm{T}$ cell agents, B cell agents and cellular therapies. Krepsova et al (16) reported that basiliximab induction may result in an increase of regulatory $\mathrm{T}$ cells and various biomarkers associated with operational tolerance expression. Additionally, long-term allograft acceptance was achieved by $B$ cell depletion treatment in kidney transplant recipients (17). Mixed chimerism, defined as cells from different donor origins coexisting in the same organism, is another form of tolerance. In 1995, Kawai et al (18) developed a non-myeloablative regimen that induced mixed chimerism and kidney allograft tolerance in major histocompatibility complex-mismatched cynomolgus monkeys. Further clinical studies have demonstrated that operational tolerance may be achieved in HLA-mismatched recipients by induction of transient chimerism via donor bone marrow transplantation (19). 
In conclusion, the present case report primarily describes the immunologic tolerance observed in a kidney transplantation recipient with minimal immunosuppressive agent induction. In the present case, although the pathological conditions of the transplanted kidney exhibited a degree of impaired kidney function, $\mathrm{T}$ or B cell biomarkers associated with immunologic tolerance were not observed; however but the patient exhibited normal kidney function. This suggests that this patient exhibited operational tolerance to some extent and that the underlying mechanism may be mixed chimerism. This condition may suggest that operational tolerance may be developed into a clinical reality when appropriate treatment is administered; however, the underlying mechanisms and biomarkers require further elucidation. The clinical application of operational tolerance may improve the prognoses of kidney transplant and other organ transplant recipients following successful immunosuppression withdrawal, and improve graft function and host defenses.

\section{Acknowledgements}

We would like to thank Dr. Hui Li from the Department of Radiology (Beijing Chao-Yang Hospital, Capital Medical University) for providing the CT images, Dr. Zexing Yu from the Department of Ultrasound (Beijing Chao-Yang Hospital, Capital Medical University) for providing the Doppler ultrasonography images, Dr. Lei Jiang from the Department of Pathology (Beijing Chao-Yang Hospital, Capital Medical University) for providing the H\&E staining images of the renopuncture biopsy.

\section{Funding}

The present study was supported by the Beijing Municipal Administration of Hospitals Clinical Medicine Development of Special Funding Support (grant no. ZYLX201408) and the China Postdoctoral Science Foundation (grant no. 2015M581131).

\section{Availability of data and materials}

The datasets used and/or analyzed during the current study are available from the corresponding author on reasonable request.

\section{Authors' contributions}

SF contributed to the study design, collection and interpretation of data, literature search, and drafting of the manuscript. YZ contributed to the collection and interpretation of data, and drafting of the manuscript. HL contributed to the study design, interpretation of data, literature search and manuscript review. $\mathrm{XZ}$ contributed to the interpretation of data, literature search, manuscript review and collection of funds.

\section{Ethics approval and consent to participate}

The present study was approved by the Ethics Committee of Beijing Chao-Yang Hospital (Capital Medical University, Beijing, China) and a written informed consent form was obtained from the patient. All procedures performed were in accordance with the ethical standards of the institutional and/or national research committee and with the 1964 Helsinki declaration and its later amendments or comparable ethical standards.

\section{Consent for publication}

Not applicable.

\section{Competing interests}

All authors have no conflict of interest to declare.

\section{References}

1. Newell KA and Turka LA: Tolerance signatures in transplant recipients. Curr Opin Organ Transplant 20: 400-405, 2015.

2. Głyda M, Czapiewski W, Karczewski M, Pięta R and Oko A: Influence of donor and recipient gender as well as selected factors on the five-year survival of kidney graft. Pol Przegl Chir 83: 188-195, 2011.

3. Marcén R: Immunosuppressive drugs in kidney transplantation: Impact on patient survival, and incidence of cardiovascular disease, malignancy and infection. Drugs 69: 2227-2243, 2009

4. Marchetti P and Navalesi R: The metabolic effects of cyclosporin and tacrolimus. J Endocrinol Invest 23: 482-490, 2000

5. Okonkwo IN, Ogbu, II, Ijoma UN, Ulasi II and Ijoma CK: Reference intervals for serum cystatin $\mathrm{C}$ and creatinine of an indigenous adult Nigerian population. Niger J Clin Pract 18: 173-177, 2015

6. Luippold G, Benöhr P, Piesch C, Heyne N and Mühlbauer B: Urinary dopamine excretion in healthy volunteers: Effect of sodium diet and acute water load. Pflugers Arch 440: 28-33, 2000.

7. Luan Y, Mosheir E, Menon MC, Wilson D, Woytovich C, Ochando J and Murphy B: Monocytic myeloid-derived suppressor cells accumulate in renal transplant patients and mediate CD4(+) Foxp3(+) Treg expansion. Am J Transpl 13: 3123-3131, 2013.

8. Owen RD: Immunogenetic consequences of vascular anastomoses between bovine twins. Science 102: 400-401, 1945.

9. Billingham RE, Brent L and Medawar PB: Actively acquired tolerance of foreign cells. Nature 172: 603-606, 1953.

10. Girmanova E, Hruba P and Viklicky O: Circulating biomarkers of tolerance. Transpl Rev (Orlando) 29: 68-72, 2015.

11. Orlando G, Hematti P, Stratta RJ, Burke GW III, Di Cocco P, Pisani F, Soker S and Wood K: Clinical operational tolerance after renal transplantation: Current status and future challenges. Ann Surg 252: 915-928, 2010.

12. Brouard S, Mansfield E, Braud C, Li L, Giral M, Hsieh SC, Baeten D, Zhang M, Ashton-Chess J, Braudeau C, et al: Identification of a peripheral blood transcriptional biomarker panel associated with operational renal allograft tolerance. Proc Natl Acad Sci USA 104: 15448-15453, 2007.

13. Heidt $\mathrm{S}$ and Wood KJ: Biomarkers of operational tolerance in solid organ transplantation. Exp Opin Med Diagn 6: 281-293, 2012.

14. Sakaguchi S: Regulatory T cells: Key controllers of immunologic self-tolerance. Cell 101: 455-458, 2000.

15. Louis S, Braudeau C, Giral M, Dupont A, Moizant F, Robillard N, Moreau A, Soulillou JP and Brouard S: Contrasting CD25hiCD4+T cells/FOXP3 patterns in chronic rejection and operational drug-free tolerance. Transplantation 81: 398-407, 2006.

16. Krepsova E, Tycova I, Sekerkova A, Wohlfahrt P, Hruba P, Striz I, Sawitzki B and Viklicky O: Effect of induction therapy on the expression of molecular markers associated with rejection and tolerance. BMC Nephrol 16: 146, 2015.

17. Kopchaliiska D, Zachary AA, Montgomery RA and Leffell MS: Reconstitution of peripheral allospecific CD19+ B-cell subsets after B-lymphocyte depletion therapy in renal transplant patients. Transplantation 87: 1394-1401, 2009.

18. Kawai T, Cosimi AB, Colvin RB, Powelson J, Eason J, Kozlowski T, Sykes M, Monroy R, Tanaka M and Sachs DH: Mixed allogeneic chimerism and renal allograft tolerance in cynomolgus monkeys. Transplantation 59: 256-262, 1995.

19. Kawai T, Sachs DH, Sprangers B, Spitzer TR, Saidman SL, Zorn E, Tolkoff-Rubin N, Preffer F, Crisalli K, Gao B, et al: Long-term results in recipients of combined HLA-mismatched kidney and bone marrow transplantation without maintenance immunosuppression. Am J Transpl 14: 1599-1611, 2014. 